\title{
Cellular retinoic acid binding protein 1 protects mice from high fat diet-induced obesity by decreasing adipocyte hypertrophy
}

\author{
Yi-Wei Lin ${ }^{1}$, Sung Wook Park ${ }^{1}$, Yu-Lung Lin ${ }^{1}$, Frank H. Burton ${ }^{1,2}$, Li-Na Wei ${ }^{1,{ }^{*}}$ \\ ${ }^{1}$ Department of Pharmacology, University of Minnesota Medical School, Minneapolis, Minnesota \\ 55455, USA. \\ ${ }^{2}$ Hennepin Healthcare Research Institute, Minneapolis, Minnesota 55415, USA
}

\begin{abstract}
Objectives-Obesity, an emerging global health issue, involves numerous factors; understanding its underlying mechanisms for prevention and therapeutics is urgently needed. Cellular retinoic acid binding protein 1 (Crabp1) knockout (CKO) mice exhibit an obese phenotype under normal diet feedings, which prompted us to propose that Crabp1 could play a role in modulating adipose tissue development/homeostasis. Studies were designed to elucidate the underlying mechanism of Crabp1's action in reducing obesity.
\end{abstract}

Subjects/methods-In animal studies, 6 weeks old male wild type and CKO mice were fed with normal diet (ND) or high fat diet (HFD) for 10 weeks. Body weight and food intake were regularly monitored. Glucose tolerance test and biological parameters of plasma (glucose and insulin levels) were measured after 10 weeks of ND vs. HFD feedings. Visceral adipose tissues were collected for histological and molecular analyses to determine affected signaling pathways. In cell culture studies, the 3T3L1 adipocyte differentiation model was used to examine and validate relevant signaling pathways.

Results-CKO mice, compared to WT mice, gained more body weight, exhibited more elevated fasting plasma glucose levels, and developed more severe impaired glucose tolerance under both ND and HFD. Histological examination revealed readily increased adipocyte hypertrophy and adipose tissue inflammation under HFD feedings. In 3T3L1 adipocytes, Crabp1 silencing enhanced extracellular signal-regulated kinase 1/2 (ERK1/2) activation, accompanied by elevated markers and signaling pathways of lipid accumulation and adipocyte hypertrophy.

Conclusions-This study identifies Crabp1's physiological role against the development of obesity. The protective function of CRABP1 is likely attributed to its classically proposed (canonical) activity as a trap for RA, which will reduce RA availability, thereby dampening RAstimulated ERK1/2 activation and adipocyte hypertrophy. The results suggest Crabp1 as a potentially new therapeutic target in managing obesity and metabolic diseases.

Users may view, print, copy, and download text and data-mine the content in such documents, for the purposes of academic research, subject always to the full Conditions of use:http://www.nature.com/authors/editorial_policies/license.html\#terms

*Correspondence: Li-Na Wei, 612 Jackson Hall, 321 Church St. SE, Minneapolis, MN 55455, Tel number: 612-625-9402, weixx009@umn.edu.

Conflicts of interest

The authors declare no conflicts of interest. 


\section{Introduction}

Obesity and its associated complications contribute to the development of type 2 diabetes and metabolic syndrome, and represent one of the most serious public health problems [13]. White adipose tissue (WAT) plays a critical role in whole body energy homeostasis. Expanding WAT mass during the development of obesity is associated with insulin resistance (IR) and related complications [4]. WAT mass expansion occurs through two major mechanisms: hyperplasia (increasing cell number) and hypertrophy (increasing cell size) [4, 5]. Improper size expansion of adipocytes results in a loss of insulin sensitivity and changes in secretary patterns of adipocyte cytokines, indicative of a pro-inflammatory stage $[3,6]$. The underlying mechanisms of adipocyte differentiation and expansion involve a group of transcriptional factors such as activating protein-1 (AP-1), peroxisome proliferatoractivated receptors gamma (PPAR $\gamma$ ), CCAAT-enhancer-binding proteins (CEBPs), krupplelike factors (KLFs) and sterol regulatory element-binding protein-1 (SREBP-1) [7]. Highly conserved signaling pathways, including $\mathrm{Wnt} / \beta$-catenin and hedgehog signaling cascades, are also involved in adipogenesis process. [8, 9]. Additionally, the extracellular signalregulated kinase 1/2 (ERK1/2) is known to promote adipocyte differentiation and hypertrophy $[10,11]$. Importantly, pharmacological treatment to reduce adipocyte hypertrophy has been shown to improve IR and resolve adipose inflammation $[10,12,13]$. Identifying factors contributing to these adipose tissue expansion and inflammatory signal pathways is important for managing obesity and associated metabolic diseases.

Cellular retinoic acid binding protein 1 (Crabp1) is a cytosolic protein with a high-affinity toward retinoic acid (RA). Classically, it is believed that Crabp1 acts to modulate free cellular RA concentration, thereby modulating RA-regulated gene expression [14, 15]. Although this is believed to be the primary function of Crabp1, recent studies have also revealed certain non-canonical activities of Crabp1, such as rapidly regulating signaling pathways like ERK1/2 [16, 17] and calcium-calmodulin protein kinase II (CaMKII) [18] signaling cascades without affecting genome activity. These non-canonical activities of Crabp1 have been demonstrated to modulate specific cellular processes in a context dependent manner, for example, in stem cell cycle control [19] and cardiomyocyte function [18].

Previously, in a 3T3L1 adipocyte differentiation model, we found that Crabp1 expression was dramatically reduced in differentiating adipocytes [20], suggesting that Crabp1 might be involved in adipocyte differentiation/expansion. Interestingly, we recently found that Crabp1 knockout (CKO) mice gained more weight than wild type mice even under normal diet (ND) feedings. We thus examined whether high-fat diet (HFD) might further affect the development of obesity in $\mathrm{CKO}$, aiming to understand the physiological role of Crabp1 in adipocyte differentiation/adipose tissue remodeling during the development of obesity and IR.

Our results presented here show that expressing Crabp1 can also prevent HFD-induced adipocyte hypertrophy, corresponding with reduced ERK1/2 activation (such as that stimulated by RA) and associated adipocyte differentiation. In this context Crabp1 exploits its classically proposed (canonical) function as an RA trap to limit RA availability, thus 
dampening ERK1/2 activity for adipocyte differentiation/hypertrophy. As such, CKO mice exhibit increased WAT hypertrophy, increased IR, and adipose inflammation. This is the first study demonstrating a new physiological role for Crabp1 in modulating adipose tissue homeostasis.

\section{Material and Methods}

\section{Animals}

Male wild type C57BL/6J mice were obtained from The Jackson Laboratory. C57BL/6J background Crabp1-null (CKO) mice were generated as described [18, 19] from a Crabp1targeted DE3 (ES) clone containing an interrupted Crabp1 allele with a 5 bp insertion. Mice were randomly divided into designated experimental groups, and 3 mice were housed in one cage. Mice were maintained in the University of Minnesota animal facility on a 14-hour light/10-hour dark cycle (lights on/off at 0600/2000) at $22 \pm 1{ }^{\circ} \mathrm{C}$ with ad-lib food and water. All animals were health before experiments and exhibited no adverse effects after experiments. Normal diet (ND) (2018; Harlan Teklad, Madison, WI) and high-fat diet (HFD) with 60\% calories from fat (F3282; Bio-Serv, West Chester, PA) were provided to animals during the experiment period. Experimental procedures were in accordance with NIH guidelines and approved by the University of Minnesota Institutional Animal Care and Use Committee.

\section{Cell culture}

The 3T3L1 cell line was a kind gift from Dr. David Bernlohr, University of Minnesota. 3T3L1 cells' adipocyte differentiation was induced by an adipogenic cocktail as described [20]. Cells were regularly tested for mycoplasma contamination. For Crabp1 silencing, 3T3L1 cells were transfected with negative- control scramble siRNA vs. Crabp1 siRNA using Hiperfect transfection reagent (Qiagen) according to manufacturer instructions. The siRNAs were transfected prior to differentiation and again on differentiation day 4 . Mouse Crabp1 siRNA sequences were $5^{\prime}$-CACGTGGGAGAATGAGAACAA-3' ${ }^{\prime}$ and $5^{\prime}$ CAGCTTGTTCCTGCTTCATGA-3'.

\section{Glucose tolerance test (GTT)}

Mice were fasted overnight and the baseline blood samples were collected. After D-glucose $(2 \mathrm{~g} / \mathrm{kg}$ ) i.p. injection, mice blood glucose levels were measured every 30 minutes with a glucometer (OneTouch Ultra).

\section{Histology}

Tissues were fixed with $10 \%$ formalin and embedded in paraffin by Comparative Pathology Shared Resource (CPSR), University of Minnesota. Paraffin-embedded tissues were sectioned and stained with hematoxylin and eosin (H\&E) for morphometric study.

\section{Western blotting}

Protein samples were isolated and processed as described [21]. Anti-phospho-ERK1/2 (catalog\# 9101, 1/1000) and anti-ERK1/2 (catalog\# 9102, 1/1000) antibodies were obtained 
from Cell Signaling. Anti- $\beta$-actin (catalog\# SC-47778, 1/1000) and anti-rabbit-IgG-HRP (catalog\# SC-2357, 1/5000) antibodies were obtained from Santa Cruz. Anti-mouse-IgGHRP (catalog\# GTX26789, 1/5000) antibody was obtained from GeneTex.

\section{RNA isolation and gene expression analysis}

Total RNA was isolated using Trizol reagent according to manufacturer instructions (Invitrogen). Complimentary DNA was obtained using High-Capacity cDNA Reverse Transcription Kit (Applied Biosystems). Quantitative real-time PCR (QPCR) was performed using Maxima SYBR Green qPCR Master Mixes (Thermo Scientific) as described previously [22]. Analysis for each gene was carried out three times and normalized to $\beta$ actin housekeeping gene expression. Primer information is provided in Supplementary Information.

\section{Oil Red $O$ staining}

Fixed cells were stained with oil red $\mathrm{O}$ as described [23]. Briefly, cells were stained with oil red $\mathrm{O}$ working solution $(0.3 \% \mathrm{w} / \mathrm{w})$ for 10 minutes. Oil red $\mathrm{O}$ was eluted by $100 \%$ isopropanol and optical density at wavelength $500 \mathrm{~nm}$ was determined for quantification.

\section{Plasma measurement}

Mice were fasted for $16 \mathrm{~h}$ for collecting fasting plasma. Blood was collected at the time of sacrifice. Fasting plasma insulin levels were measured using an insulin ELISA kit according to manufacturer's instruction (catalog number EMINS; ThermoFisher Scientific).

\section{Statistical analysis}

Sample size for animal experiments were determined based on previous studies using HFD mouse models. No animals were excluded from the analyses. The investigators were not blinded to the group allocation during the experiment. Two-way ANOVA or unpaired twotailed Student's $t$-test was used when appropriate for comparison among groups. Data were normal distributed and variance was similar between the groups that are being statistically compared. Data were presented as means \pm SEM. The comparison was considered statistically significant when $p$ values $\leq 0.05(* p<0.05 ; * *<0.01 ; * * * p<0.001)$.

\section{Results}

\section{CKO mice gained more weight and developed diabetes complications.}

Previously, our laboratory found that during adipocyte differentiation in vitro, Crabp1 expression was initially up-regulated in 3T3L1 preadipocytes, but then the gene was silenced via heterochromatin formation when cultures were undergoing adipocyte differentiation [20]. The tight regulation of Crabp1 gene expression suggested that Crabp1 might play a role in regulating adipocyte differentiation. Given that adipose tissue is the primary contributor to the development of obesity, we then monitored the phenotype of CKO mice under ND and HFD feedings for a comparison to wild type mice. We found that CKO mice gained more body weight under both HFD and ND feedings (Fig. 1a). On average, WT mouse gained approximately $3 \mathrm{~g}$ during the 5 weeks of monitoring; whereas under the same 
experimental condition, CKO mouse gained approximately $4 \mathrm{~g}$. Even in ND, CKO mice readily gained more body weight at the age of 12 weeks. Careful weekly monitoring of diet consumption revealed that CKO mice consumed more food than wild type mice (Fig. 1b). We noticed that our measurements of food intake $(0.25 \mathrm{~g} / \mathrm{g}$ vs. $0.3 \mathrm{~g} / \mathrm{g}$ for WT vs. CKO) might have slightly over-estimated the food consumed $(0.2 \mathrm{~g} / \mathrm{g}$ reported); this could be due to food dropped to the bedding without being consumed. Nevertheless, under the same experimental conditions, the difference in the amounts of food consumed by WT vs. CKO mice was obvious and statistically significant. We then monitored CKO mice for features of type 2 diabetes. First, GTT, performed at 10 weeks following HFD feedings, showed significant glucose intolerance in CKO mice, but there was no impaired islet insulin secretion (Fig. 1c, Fig. S1). Further, CKO mice presented higher fasting plasma glucose levels (Fig. 1d). These results showed that CKO mice under ND feedings readily developed obesity, and under HFD feedings further developed severe diabetes.

\section{CKO mice presented WAT hypertrophy and inflammation.}

Given that CKO mice were demonstrably prone to develop obesity and diabetes, we thus investigated their adipose tissues, especially WAT. We found that CKO mice gained more WAT mass under both ND and HFD feedings (Fig. 2a). Histological analysis of WAT revealed expanded adipocytes (increase in adipocyte size) and inflammation (Fig. 2b, arrows). We scored the adipocyte number within the same area between WT and CKO, which showed correspondingly reduced cell number in CKO tissue (Fig. 2b lower left panels) that exhibited apparent hypertrophy (Fig $2 \mathrm{~b}$ lower right panels). However, we could not yet rule out the possibility of hyperplasia contributing to adipose expansion in CKO mice. This indicated that CKO mice's WAT was more inflamed and exhibited hypertrophy. This hypertrophy phenotype was further validated in gene expression analyses of mesoderm specific transcript (Mest) and secreted frizzled related protein 5 (Sfrp5) (Fig. 2c) [24, 25]. Additional gene and metabolic analyses showed that the WAT of CKO mice, especially under HFD feedings, also accumulated more fat (indicated by increased perilipin 1 expression in CKO/HFD as compared to WT/HFD WAT) and consumed less energy (indicated by decreased uncoupling protein $1[U C P-1]$ expression in CKO/HFD as compared to WT/HFD WAT) (Fig. 2c).

\section{Silencing Crabp1 led to fat accumulation and hypertrophy in adipocyte cultures.}

The hypertrophic obese adipose phenotype of CKO mice suggests a dampening effect of Crabp1 upon adipose tissue formation. This is consistent with our previous finding, in an in vitro adipocyte differentiation model, of significantly decreased Crabp1 expression during adipocyte differentiation [20]. To validate the specific functional role of Crabp1 as a negative factor in adipocyte differentiation, we again employed this well-established 3T3L1 adipocyte differentiation model, but now using gene silencing. Crabp1-specific siRNAs were used to silence Crabp1 expression in preadipocyte cultures that were then induced for adipocyte differentiation. The results showed that silencing Crabp1 indeed increased fat accumulation in the later stage of adipocyte differentiation (Fig. 3a). Consistently, not only perilipin- 1 but also Cebpa, Cebp $\beta$, and fatty acid binding protein 4 (Fabp4) adipocytemarker genes all were expressed at higher levels in fully differentiated adipocytes when Crabp1 is silenced (Fig. 3b). 
Moreover, silencing Crabp1 led to higher levels of adipose-cell hypertrophy markers (Mest and Sfrp5) (Fig. 3c). Mechanistically, we found that silencing Crabp1 resulted in specifically elevated ERK1/2 signaling, as indicted in increased pERK1/2 signal (Fig. 3d, top panels), which is known to promote both adipocyte hypertrophy and adipogenesis $[10,11]$. In multiple classical studies reported earlier, ERK1/2 was known to be activated (and elevated) by canonical RA signaling [26, 27], while Crabp1 was known to reduce RA availability for its canonical gene regulatory actions including to elevate ERK1/2 signaling [15]. Therefore, silencing Crabp1 would increase RA availability, thereby enhancing ERK1/2 signaling and consequent adipogenesis and adipocyte hypertrophy. All together, this present study elucidates a new biological role for Crabp1 -- to negatively modulate adipocyte differentiation, and especially to block adipose hypertrophy and adipogenesis. Deleting Crabp1 thus enhances animals' vulnerability to HFD-induced adipose hypertrophy and adipogenesis.

\section{Discussion}

RA, when exogenously administered pharmacologically, has been shown to inhibit, rather than induce, adipogenesis to suppress obesity in both cell and animal models [28-32]. One of the underlying mechanisms by which RA does so is by acting on PPARs and RA receptors (RARs). Pharmacological RA was also reported to activate Wnt signaling to inhibit adipogenesis [33]. Zfp423 transcription factor has been reported to be involved in anti-adipogenesis activity of RA [34]. RA is also known to balance adipose tissue immunehomeostasis, including regulating nuclear factor kappa-light-chain-enhancer of activated $\mathrm{B}$ cells $(\mathrm{NFkB})$ signaling, macrophage polarization, and regulatory $\mathrm{T}$ cell (Treg) polarization [35-38]. In contrast, findings have also been reported that RA can promote adipogenesis $[39,40]$. These studies consistently suggested multiple pathways mediate such opposing actions of RA. However, because most of these studies applied RA as a pharmacological agent, the relevance of physiological RA, and of components like Crabp1 that regulate RA's physiological availability, remained entirely unclear.

This current study employed a knockout mouse model aiming to determine the relevance of physiological RA by specifically examining the role of one of its metabolic regulators, Crabp1, that's long been suggested to reduce its physiological availability. The enhanced vulnerability of the Crabp1-knockout "CKO" mice to both ND-associated and HFD-super induced adipose hypertrophy and adipogenesis indicates, first, that RA enhances the ultimate development of adipose tissues, and Crabp1 potentially serves as a "physiological resistor" that limits RA's availability. If or when RA levels accidentally surge, Crabp1 will decrease any tendency to overtly acquire adiposity (adipose expansion). To this end, literature has reported that another RA binding protein, Crabp2, is repressed in early preadipocyte/ adipocyte differentiation. With this observation, the author suggested that down-regulating Crabp2 in preadipocyte would blunt RA's early suppressive effect on adipogenesis [41]. Thus, an interesting possibility exists for a functional connection between Crabp1 and Crabp2 in maintaining adipose tissue homeostasis, such as to reduce RA's availability for adipose expansion (via expressing Crabp1) and to suppress RA's nuclear signaling (via reducing Crabp2) that limits early adipocyte differentiation. Validation of this interesting possibility will require further detailed studies in a physiological context. 
Among all members of RA-metabolizing or signaling pathways, Crabp1 is the most conserved protein. For instance, between mouse and human Crabp1's, there is only one amino acid substitution in its entire coding region [14]. The high evolutionary conservation of this protein suggests an important physiological role for Crabp1 that benefits the survival or procreative well-being of both mice and men. Previous gene knockout studies reported that CKO mice seemed to exhibit no apparent phenotype under a normal laboratory housing condition $[42,43]$, which we assert may have led to underestimating the potential physiological importance of Crabp1. In our present study to examine the intersection between Crabp1 loss and diet in these CKO mutant mice, we uncovered a new physiological role for Crabp1 specifically in modulating adipose homeostasis. Previously, we have reported that young CKO mice ( 2 months old), while appearing normal in laboratories, exhibit more severe heart damage when mice are subjected to cardiac stress induction [18], indicating that Crabp1 serves also as a "physiological resistor" in the face of potential heart damages -- a cardiac protectant against catecholamine-induced damage. But in this case, the affected signaling pathway is CaMKII. These studies suggest there are multiple physiological functions and potential associated signaling pathways of Crabp1, and that they are likely to be cell/tissue context-dependent. More whole animal physiological studies are needed in order to uncover other potential physiological roles of Crabp1.

Proper WAT expansion and adipocyte differentiation provide a protective mechanism to store excess lipid [44]. However, nutrient overload leads to improper WAT expansion, resulting in hypoxia and inflammation especially in WAT [4]. These pathological features of WAT occur during the development of obesity, and can further lead to IR and related complications [4]. In this study, we found not only enhanced adipogenesis but also increased adipocyte hypertrophy in CKO mice. We also observed increased WAT inflammation (macrophage infiltration) in CKO mice (Fig. 2b). However, because Crabp1 expression in macrophages is very low, it doesn't seem to have a direct effect in macrophage differentiation or maturation. It thus may be interesting to examine if CKO adipocytes could secrete certain adipokines that modulate macrophage infiltration and/or polarization.

Adipocyte differentiation is a highly regulated process that involves not only multiple sets of transcriptional factors but also various signaling pathways. Moreover, endocrine factors such as thyroid hormone and sterol hormones also critically control adipogenesis [7]. Studies have demonstrated that thyroid hormones regulate adipocyte development and are required for adipogenesis $[45,46]$. Our previous studies demonstrated that thyroid hormones regulate the expression of Crabp1 in a biphasic manner. In pre-adipocytes, thyroid hormones first elevate the expression of Crabp1 [47]. Subsequently, thyroid hormones then repress the expression of Crabp1 during adipocyte differentiation through inducing heterochromatin formation of this gene and its complete silencing in differentiated adipocytes [20].

Consistently, results of our present study confirm that Crabp1 is not a desired component for, but rather is a negative modulator of, adipocyte differentiation.

ERK has been shown to promote adipogenesis in various in vitro models [11, 48, 49], as well as in embryonic stem cells committed to adipocyte lineage [27]. It has also been reported that ERK1 knockout mice are resistant to diet-induced obesity [50]. Inhibition of ERK activity, using potent MEK inhibitors U0126 and PD98059, likewise impedes 
adipocyte differentiation [27, 51]. Recent studies also demonstrated that using chemicals that target the ERK signaling pathway effectively ameliorated obesity and IR by reversing WAT dysfunction and hypertrophy. In our current study, we likewise show that Crabp1 dampens ERK activation and its downstream signaling, leading to reduced adipogenesis and adipocyte hypertrophy. Accordingly, we've diagrammed a proposed physiological pathway for Crabp1's negative modulation of RA- and ERK-dependent adipogenesis and hypertrophy (Fig. 4). In this model, Crabp1 would employ its classically proposed (canonical) activity, which is to limit RA availability. This would reduce ERK1/2 signaling and subsequent adipocyte hypertrophy. With this model, we suggest that Crabp1 may also provide a potential therapeutic target in managing obesity or related metabolic diseases, although future physiological studies will be needed to elucidate such potential.

\section{Supplementary Material}

Refer to Web version on PubMed Central for supplementary material.

\section{Acknowledgements}

The authors thank Neal Mukherjee for technical support.

Funding

This work was supported by NIH grants DK54733, DK60521, and the Dean's Commitment and the Distinguished McKnight Professorship of University of Minnesota to LNW.

\section{References}

1. Dandona P, Aljada A, Chaudhuri A, Mohanty P, Garg R. Metabolic syndrome: a comprehensive perspective based on interactions between obesity, diabetes, and inflammation. Circulation 2005; 111(11): 1448-1454. [PubMed: 15781756]

2. Nolan CJ, Damm P, Prentki M. Type 2 diabetes across generations: from pathophysiology to prevention and management. Lancet 2011; 378(9786): 169-181. [PubMed: 21705072]

3. Donath MY, Shoelson SE. Type 2 diabetes as an inflammatory disease. Nature reviews. Immunology 2011; 11(2): 98-107.

4. Guilherme A, Virbasius JV, Puri V, Czech MP. Adipocyte dysfunctions linking obesity to insulin resistance and type 2 diabetes. Nat Rev Mol Cell Biol 2008; 9(5): 367-377. [PubMed: 18401346]

5. Jo J, Gavrilova O, Pack S, Jou W, Mullen S, Sumner AE et al. Hypertrophy and/or Hyperplasia: Dynamics of Adipose Tissue Growth. PLoS Comput Biol 2009; 5(3): e1000324. [PubMed: 19325873]

6. Esser N, Legrand-Poels S, Piette J, Scheen AJ, Paquot N. Inflammation as a link between obesity, metabolic syndrome and type 2 diabetes. Diabetes Res Clin Pract 2014; 105(2): 141-150. [PubMed: 24798950]

7. Sarjeant K, Stephens JM. Adipogenesis. Cold Spring Harb Perspect Biol 2012; 4(9): a008417. [PubMed: 22952395]

8. Rosen ED, MacDougald OA. Adipocyte differentiation from the inside out. Nat Rev Mol Cell Biol 2006; 7(12): 885-896. [PubMed: 17139329]

9. Chen N, Wang J. Wnt/beta-Catenin Signaling and Obesity. Front Physiol 2018; 9: 792. [PubMed: 30065654]

10. Ozaki KI, Awazu M, Tamiya M, Iwasaki Y, Harada A, Kugisaki S et al. Targeting the ERK signaling pathway as a potential treatment for insulin resistance and type 2 diabetes. American journal of physiology. Endocrinology and metabolism 2016; 310(8): E643-E651. [PubMed: 26860984] 
11. Prusty D, Park BH, Davis KE, Farmer SR. Activation of MEK/ERK signaling promotes adipogenesis by enhancing peroxisome proliferator-activated receptor gamma (PPARgamma) and $\mathrm{C} / \mathrm{EBPalpha}$ gene expression during the differentiation of 3T3-L1 preadipocytes. The Journal of biological chemistry 2002; 277(48): 46226-46232. [PubMed: 12270934]

12. Murtaza M, Khan G, Aftab MF, Afridi SK, Ghaffar S, Ahmed A et al. Cucurbitacin E reduces obesity and related metabolic dysfunction in mice by targeting JAK-STAT5 signaling pathway. PloS one 2017; 12(6): e0178910. [PubMed: 28598969]

13. Chan PC, Hsiao FC, Chang HM, Wabitsch M, Hsieh PS. Importance of adipocyte cyclooxygenase-2 and prostaglandin E2-prostaglandin E receptor 3 signaling in the development of obesity-induced adipose tissue inflammation and insulin resistance. FASEB journal : official publication of the Federation of American Societies for Experimental Biology 2016; 30(6): 2282 2297. [PubMed: 26932930]

14. Fiorella PD, Giguere V, Napoli JL. Expression of cellular retinoic acid-binding protein (type II) in Escherichia coli. Characterization and comparison to cellular retinoic acid-binding protein (type I). The Journal of biological chemistry 1993; 268(29): 21545-21552. [PubMed: 8408005]

15. Wei LN, Chang L, Hu X. Studies of the type I cellular retinoic acid-binding protein mutants and their biological activities. Mol Cell Biochem 1999; 200(1-2): 69-76. [PubMed: 10569185]

16. Persaud SD, Lin YW, Wu CY, Kagechika H, Wei LN. Cellular retinoic acid binding protein I mediates rapid non-canonical activation of ERK1/2 by all-trans retinoic acid. Cellular signalling 2013; 25(1): 19-25. [PubMed: 22982089]

17. Persaud SD, Park SW, Ishigami-Yuasa M, Koyano-Nakagawa N, Kagechika H, Wei LN. All transretinoic acid analogs promote cancer cell apoptosis through non-genomic Crabp1 mediating ERK1/2 phosphorylation. Scientific reports 2016; 6: 22396. [PubMed: 26935534]

18. Park SW, Persaud SD, Ogokeh S, Meyers TA, Townsend D, Wei LN. CRABP1 protects the heart from isoproterenol-induced acute and chronic remodeling. J Endocrinol 2018; 236(3): 151-165. [PubMed: 29371236]

19. Lin YL, Persaud SD, Nhieu J, Wei LN. Cellular Retinoic Acid-Binding Protein 1 Modulates Stem Cell Proliferation to Affect Learning and Memory in Male Mice. Endocrinology 2017; 158(9): 3004-3014. [PubMed: 28911165]

20. Park SW, Huang WH, Persaud SD, Wei LN. RIP140 in thyroid hormone-repression and chromatin remodeling of Crabp1 gene during adipocyte differentiation. Nucleic Acids Res 2009; 37(21): 7085-7094. [PubMed: 19778926]

21. Lin YW, Lee B, Liu PS, Wei LN. Receptor-Interacting Protein 140 Orchestrates the Dynamics of Macrophage M1/M2 Polarization. Journal of innate immunity 2016; 8(1): 97-107. [PubMed: 26228026]

22. Lin YW, Montassier E, Knights D, Wei LN. Gut microbiota from metabolic disease-resistant, macrophage-specific RIP140 knockdown mice improves metabolic phenotype and gastrointestinal integrity. Scientific reports 2016; 6: 38599. [PubMed: 27929078]

23. Lin YW, Liu PS, Adhikari N, Hall JL, Wei LN. RIP140 contributes to foam cell formation and atherosclerosis by regulating cholesterol homeostasis in macrophages. Journal of molecular and cellular cardiology 2015; 79: 287-294. [PubMed: 25528964]

24. Takahashi M, Kamei Y, Ezaki O. Mest/Peg1 imprinted gene enlarges adipocytes and is a marker of adipocyte size. American journal of physiology. Endocrinology and metabolism 2005; 288(1): E117-124. [PubMed: 15353408]

25. Mori H, Prestwich TC, Reid MA, Longo KA, Gerin I, Cawthorn WP et al. Secreted frizzled-related protein 5 suppresses adipocyte mitochondrial metabolism through WNT inhibition. The Journal of clinical investigation 2012; 122(7): 2405-2416. [PubMed: 22728933]

26. Yen A, Roberson MS, Varvayanis S, Lee AT. Retinoic acid induced mitogen-activated protein (MAP)/extracellular signal-regulated kinase (ERK) kinase-dependent MAP kinase activation needed to elicit HL-60 cell differentiation and growth arrest. Cancer Res 1998; 58(14): 31633172. [PubMed: 9679985]

27. Bost F, Caron L, Marchetti I, Dani C, Le Marchand-Brustel Y, Binetruy B. Retinoic acid activation of the ERK pathway is required for embryonic stem cell commitment into the adipocyte lineage. The Biochemical journal 2002; 361(Pt 3): 621-627. [PubMed: 11802792] 
28. Murray T, Russell TR. Inhibition of adipose conversion in 3T3-L2 cells by retinoic acid. J Supramol Struct 1980; 14(2): 255-266. [PubMed: 6164877]

29. Kamei Y, Kawada T, Mizukami J, Sugimoto E. The prevention of adipose differentiation of 3T3-L1 cells caused by retinoic acid is elicited through retinoic acid receptor alpha. Life Sci 1994; 55(16): PL307-312. [PubMed: 7934625]

30. Jeyakumar SM, Vajreswari A, Sesikeran B, Giridharan NV. Vitamin A supplementation induces adipose tissue loss through apoptosis in lean but not in obese rats of the WNIN/Ob strain. J Mol Endocrinol 2005; 35(2): 391-398. [PubMed: 16216918]

31. Berry DC, Noy N. All-trans-retinoic acid represses obesity and insulin resistance by activating both peroxisome proliferation-activated receptor beta/delta and retinoic acid receptor. Molecular and cellular biology 2009; 29(12): 3286-3296. [PubMed: 19364826]

32. Berry DC, DeSantis D, Soltanian H, Croniger CM, Noy N. Retinoic acid upregulates preadipocyte genes to block adipogenesis and suppress diet-induced obesity. Diabetes 2012; 61(5): 1112-1121. [PubMed: 22396202]

33. Kim DM, Choi HR, Park A, Shin SM, Bae KH, Lee SC et al. Retinoic acid inhibits adipogenesis via activation of Wnt signaling pathway in 3T3-L1 preadipocytes. Biochem Biophys Res Commun 2013; 434(3): 455-459. [PubMed: 23583383]

34. Wang B, Fu X, Zhu MJ, Du M. Retinoic acid inhibits white adipogenesis by disrupting GADD45A-mediated Zfp423 DNA demethylation. J Mol Cell Biol 2017; 9(4): 338-349. [PubMed: 28992291]

35. Erkelens MN, Mebius RE. Retinoic Acid and Immune Homeostasis: A Balancing Act. Trends in immunology 2017; 38(3): 168-180. [PubMed: 28094101]

36. Karkeni E, Bonnet L, Astier J, Couturier C, Dalifard J, Tourniaire F et al. All-trans-retinoic acid represses chemokine expression in adipocytes and adipose tissue by inhibiting NF-kappaB signaling. The Journal of nutritional biochemistry 2017; 42: 101-107. [PubMed: 28157617]

37. Coombes JL, Siddiqui KR, Arancibia-Carcamo CV, Hall J, Sun CM, Belkaid Y et al. A functionally specialized population of mucosal CD103+ DCs induces Foxp3+ regulatory T cells via a TGF-beta and retinoic acid-dependent mechanism. J Exp Med 2007; 204(8): 1757-1764. [PubMed: 17620361]

38. Gundra UM, Girgis NM, Gonzalez MA, San Tang M, Van Der Zande HJP, Lin JD et al. Vitamin A mediates conversion of monocyte-derived macrophages into tissue-resident macrophages during alternative activation. Nature immunology 2017; 18(6): 642-653. [PubMed: 28436955]

39. Safonova I, Darimont C, Amri EZ, Grimaldi P, Ailhaud G, Reichert U et al. Retinoids are positive effectors of adipose cell differentiation. Molecular and cellular endocrinology 1994; 104(2): 201211. [PubMed: 7988747]

40. Safonova I, Reichert U, Shroot B, Ailhaud G, Grimaldi P. Fatty acids and retinoids act synergistically on adipose cell differentiation. Biochem Biophys Res Commun 1994; 204(2): 498504. [PubMed: 7980506]

41. Berry DC, Soltanian H, Noy N. Repression of cellular retinoic binding protein II during adipocyte differentiation. The journal of biological chemistry 2010; 285:15324-15332. [PubMed: 20228061]

42. Gorry P, Lufkin T, Dierich A, Rochette-Egly C, Decimo D, Dolle P et al. The cellular retinoic acid binding protein I is dispensable. Proceedings of the National Academy of Sciences of the United States of America 1994; 91(19): 9032-9036. [PubMed: 8090764]

43. de Bruijn DR, Oerlemans F, Hendriks W, Baats E, Ploemacher R, Wieringa B et al. Normal development, growth and reproduction in cellular retinoic acid binding protein-I (CRABPI) null mutant mice. Differentiation 1994; 58(2): 141-148. [PubMed: 7890139]

44. Scherer PE. Adipose tissue: from lipid storage compartment to endocrine organ. Diabetes 2006; 55(6): 1537-1545. [PubMed: 16731815]

45. Mariash CN. Thyroid hormone and the adipocyte. J Clin Endocrinol Metab 2003; 88(12): $5603-$ 5604. [PubMed: 14671139]

46. Obregon MJ. Adipose tissues and thyroid hormones. Front Physiol 2014; 5: 479. [PubMed: 25566082] 
47. Wei LN, Hu X. Receptor interacting protein 140 as a thyroid hormone-dependent, negative coregulator for the induction of cellular retinoic acid binding protein I gene. Molecular and cellular endocrinology 2004; 218(1-2): 39-48. [PubMed: 15130509]

48. Aubert J, Dessolin S, Belmonte N, Li M, McKenzie FR, Staccini L et al. Leukemia inhibitory factor and its receptor promote adipocyte differentiation via the mitogen-activated protein kinase cascade. The Journal of biological chemistry 1999; 274(35): 24965-24972. [PubMed: 10455174]

49. Belmonte N, Phillips BW, Massiera F, Villageois P, Wdziekonski B, Saint-Marc P et al. Activation of extracellular signal-regulated kinases and CREB/ATF-1 mediate the expression of CCAAT/ enhancer binding proteins beta and -delta in preadipocytes. Mol Endocrinol 2001; 15(11): 20372049. [PubMed: 11682632]

50. Bost F, Aouadi M, Caron L, Even P, Belmonte N, Prot M et al. The extracellular signal-regulated kinase isoform ERK1 is specifically required for in vitro and in vivo adipogenesis. Diabetes 2005; 54(2): 402-411. [PubMed: 15677498]

51. Tang QQ, Otto TC, Lane MD. Mitotic clonal expansion: a synchronous process required for adipogenesis. Proceedings of the National Academy of Sciences of the United States of America 2003; 100(1): 44-49. [PubMed: 12502791] 
a

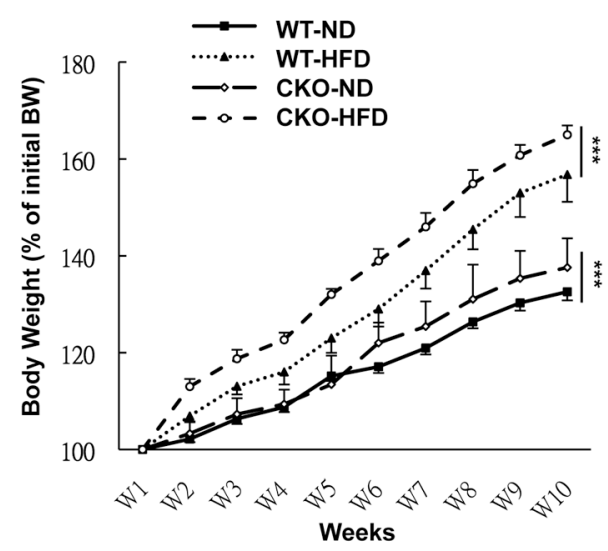

b

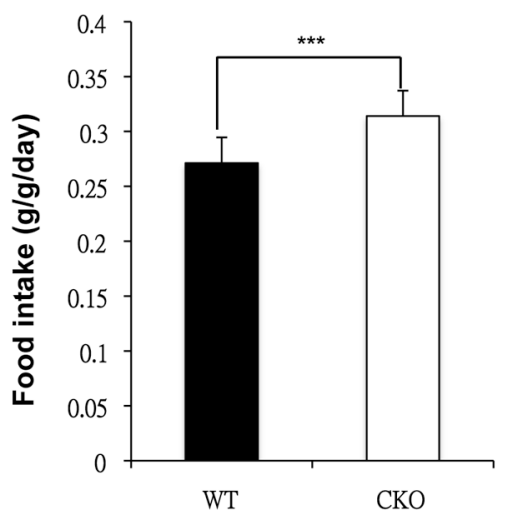

C

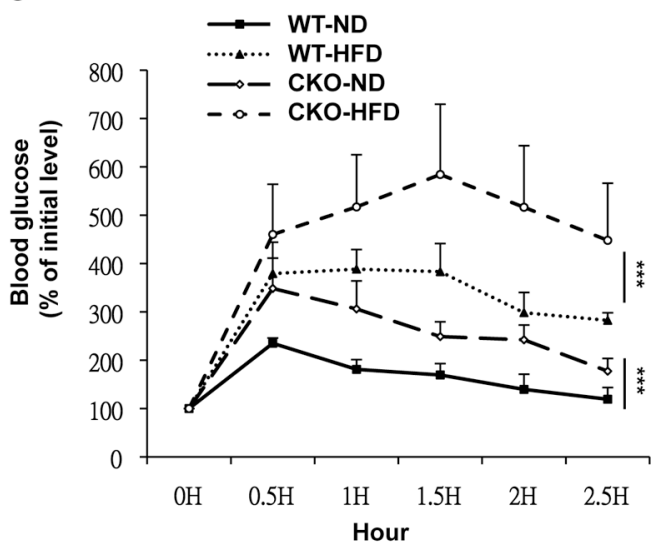

d

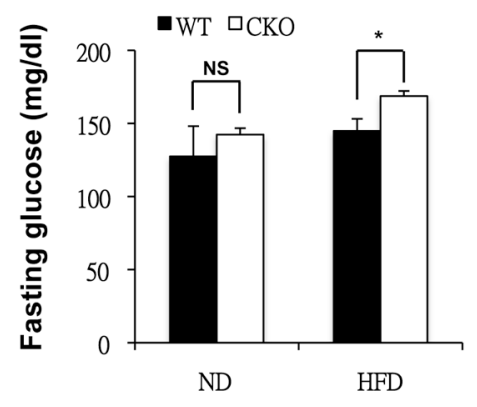

Fig. 1. CKO mice gained more weight and developed insulin resistance.

(a) Average body weight of WT and CKO mice fed a ND and HFD for 10 weeks; $n=12$ in WT-ND group; $n=6$ in WT-HFD, CKO-ND and CKO-HFD group. (b) Average body weightnormalized daily food consumption (g/g/day) of ND-fed mice; $\mathrm{n}=6$ mice per group. (c) GTT determined after 10 weeks of ND or HFD feeding; $n=6$ mice per group. (d) Fasting plasma glucose levels in ND- or HFD-fed WT and CKO mice; $n=6$ mice per group. Results are presented as mean $\pm \mathrm{S} * \mathrm{p}<0.05, * * \mathrm{p}<0.01 ; * * * \mathrm{p}<0.001$ compared to WT group. 
a

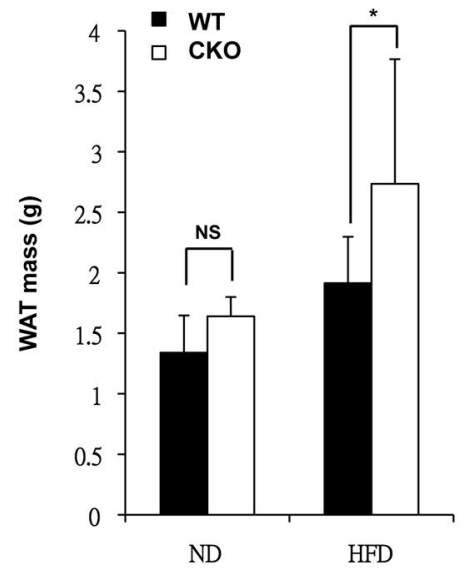

C

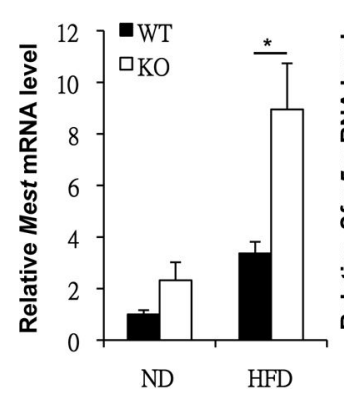

b
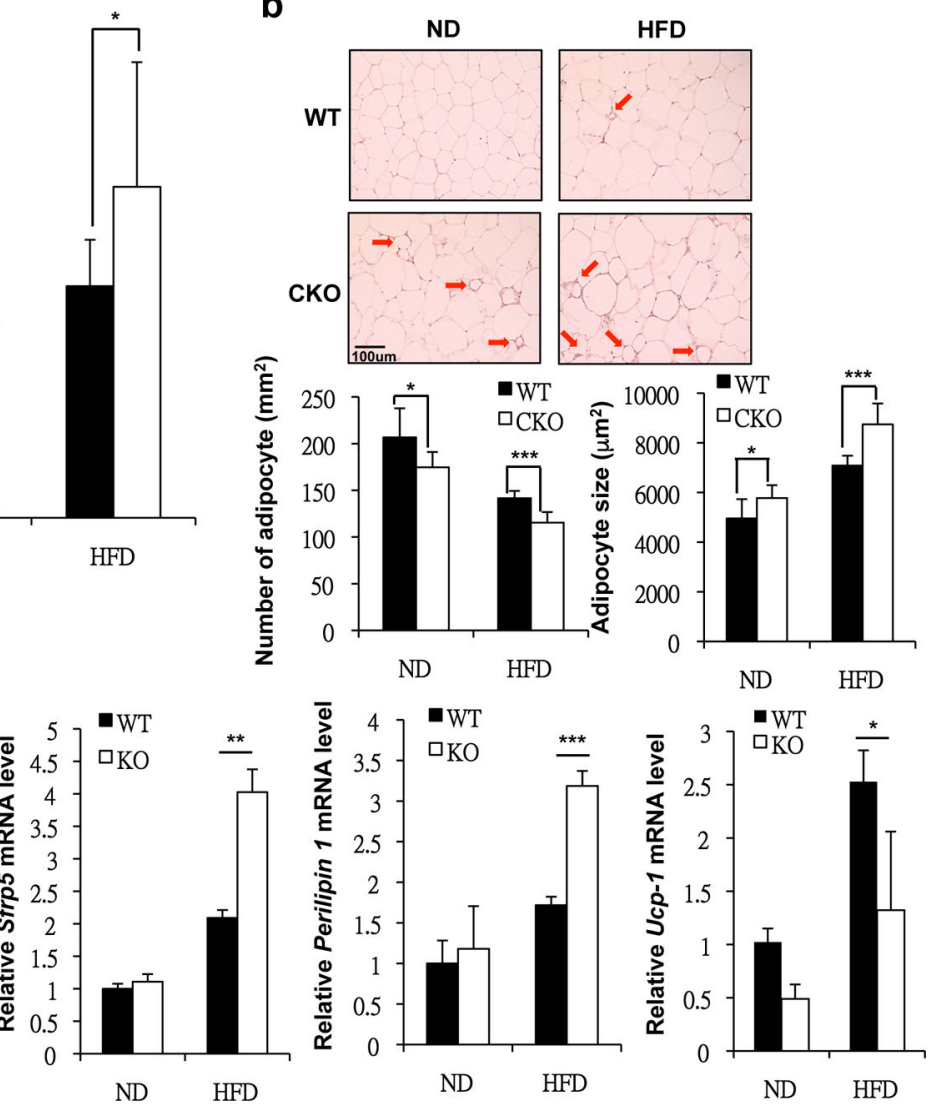

Fig. 2. CKO mice presented WAT hypertrophy and inflammation.

(a) Average visceral WAT (vWAT) tissue mass of ND- or HFD-fed WT and CKO mice. (b) Top, H\&E staining of vWAT of ND- or HFD-fed WT and CKO mice. Scale bar: $100 \mu \mathrm{m}$. Bottom, adipocytes per square millimeter and average adipocyte size of ND- or HFD-fed WT and CKO mice. (c) qPCR of Mest, Srfp5, Perilipin 1 and Ucp- 1 mRNA levels in vWAT. Results are presented as mean \pm SE; $\mathrm{n}=6$ mice per group. $* \mathrm{p}<0.05, * * \mathrm{p}<0.01 ; * * * \mathrm{p}<$ 0.001 compared to WT group. 


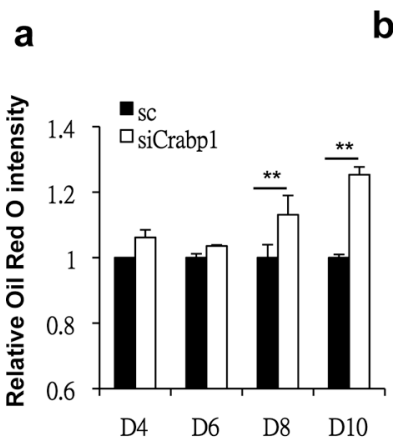

b
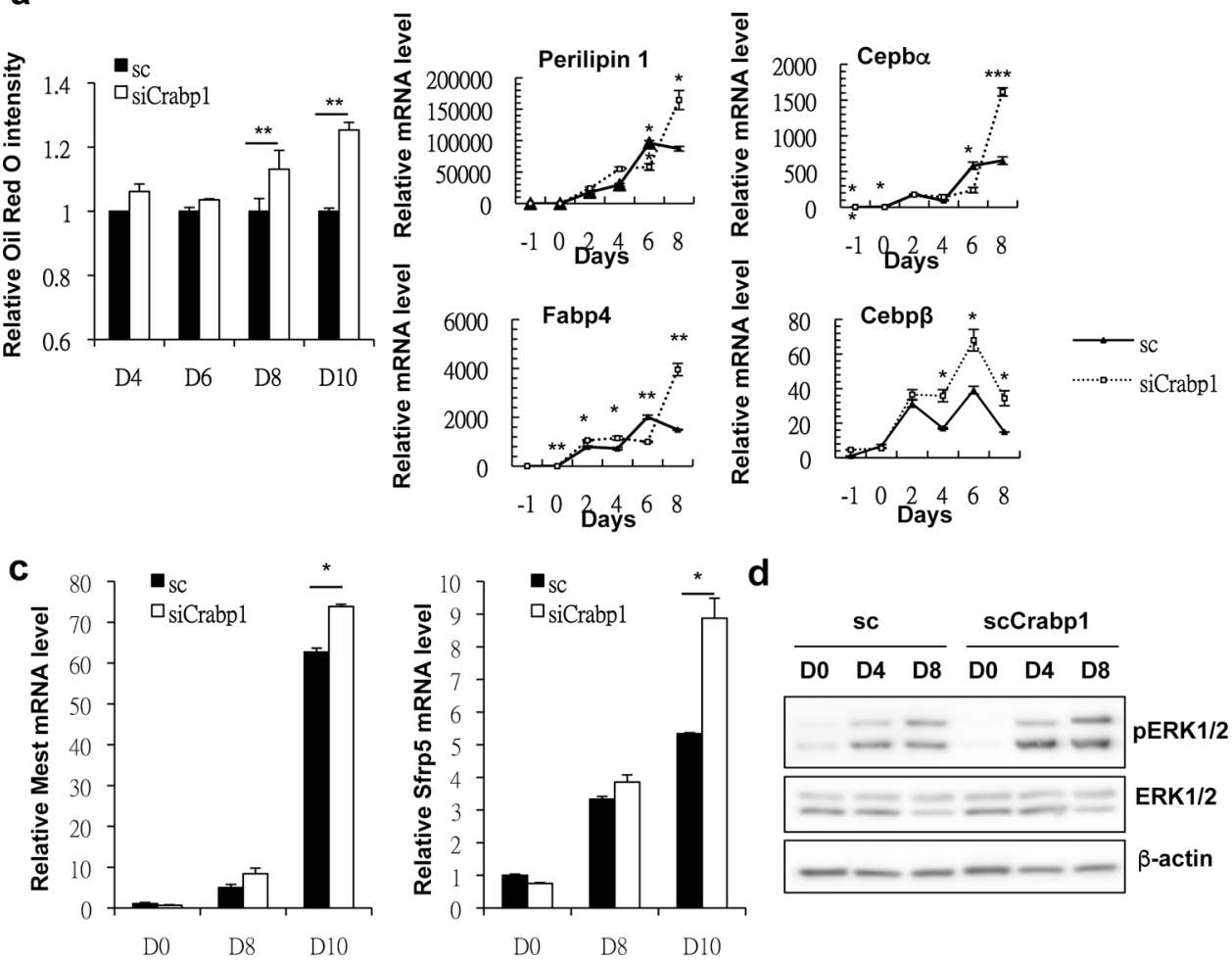

Fig. 3. Silencing Crabp1 led to fat accumulation and hypertrophy in adipocytes.

(a) Oil red $\mathrm{O}$ staining of 3T3L1 differentiated adipocytes. Results are presented as oil red $\mathrm{O}$ intensity (optical density) normalized to the sc D0 group. (b) qPCR of Perilipin 1, Cebpa, and Fabp 4 mRNA levels. (c) qPCR of Mest and Sfrp 5 mRNA levels. (d) Western blots of phospho ERK1/2 (pEKR1/2), total ERK1/2 and $\beta$-actin protein levels. All experimental data were collected from 3T3L1-differentiated adipocytes with or without silencing Crabp1 on designated days. Results are presented as mean \pm SE. $* p<0.05, * * p<0.01 ; * * p<0.001$ compared to sc group; sc, scramble control; siCrabp1, silencing Crabp1. 


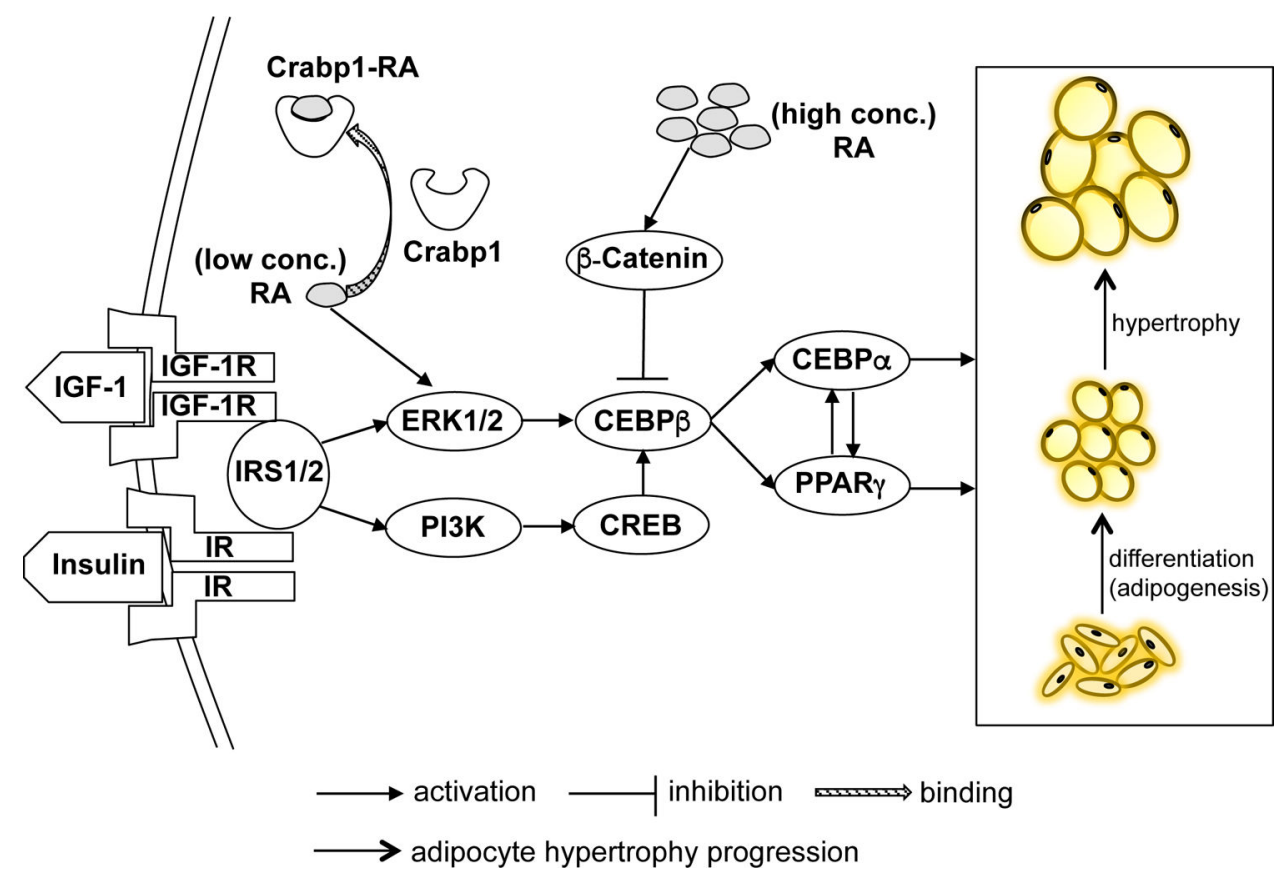

Fig. 4. Proposed mechanism of the suppressive effects of Crabp1 in adipogenesis and adipocyte hypertrophy.

Pharmacological concentrations of RA (high conc.) inhibit adipocyte adipogenesis through $\beta$-catenin pathway to dampen CEBP $\beta$ activities. On the other hand, physiological concentrations of RA (low conc.) activate ERK1/2 signaling, promoting CEBP $\beta$ activities to trigger adipocyte adipogenesis and hypertrophy. Crabp1 binds RA to reduce free RA availability, thereby dampening ERK1/2 activation and leading to decreased adipogenic signaling. Deleting Crabp1 in CKO would increase RA available to tissues, resulting in enhanced ERK1/2 signaling and its downstream adipogenic signaling. This increases animals' vulnerability to diet-induced obesity. According to this model, Crabp1 protects mice from developing obesity by reducing the adipogenesis and adipocyte hypertrophy. 